\title{
SHP2 promotes laryngeal cancer growth through the Ras/Raf/Mek/Erk pathway and serves as a prognostic indicator for laryngeal cancer
}

\author{
JIA GU $^{1 *}$, TAO HAN ${ }^{2 *}$, RUI-HANG MA ${ }^{3 *}$, YU-LIN ZHU ${ }^{1}$, YI-NA JIA ${ }^{1}$, JING-JING DU $^{1}$, \\ YU CHEN $^{1}$, XUE-JUN JIANG ${ }^{1}$, XIAO-DONG XIE ${ }^{2}$ and XING GUO ${ }^{1}$ \\ ${ }^{1}$ Department of Otolaryngology, The First Affiliated Hospital of China Medical University, \\ Shenyang 110001; Departments of ${ }^{2}$ Oncology and ${ }^{3}$ Operation Room, \\ General Hospital of Shenyang Military Region, Shenyang 110016, P.R. China
}

Received September 24, 2013; Accepted November 5, 2013

DOI: 10.3892/ijo.2013.2191

\begin{abstract}
The overall survival rate and prognosis of patients with laryngeal cancer are not optimistic despite advances in therapeutic techniques. Gene expression prognostic models enable the development of more appropriate treatment strategies. The human gene PTPN11 encoding a non-receptor protein tyrosine phosphatase, Src homology phosphotyrosine phosphatase 2 (SHP2), is a well-documented proto-oncogene in various malignancies. This study investigated the role of SHP2 expression and associated clinical manifestations in laryngeal cancer using a tissue microarray of 112 pairs of laryngeal cancer samples and corresponding adjacent normal mucosae. SHP2 expression increased in laryngeal cancer, and this result was associated with the poor survival rate of laryngeal cancer patients. Moreover, increased SHP2 expression remarkably promoted the growth of laryngeal cancer cells in vitro and tumorigenicity of laryngeal cancer cells in vivo. The Ras/Raf/Mek/Erk pathway was also found to be involved in the SHP2-induced growth of laryngeal cancer cells. Overall, our findings indicated that SHP2 plays an important role in laryngeal cancer tumorigenesis and that its expression is negatively correlated with the prognosis of patients. Thus, SHP2 may be a promising combinational therapeutic target for treatment of laryngeal cancer. The
\end{abstract}

Correspondence to: Professor Xing Guo, Department of Otolaryngology, The First Affiliated Hospital of China Medical University, 155 North Nanjing Street, Shenyang 110001, P.R. China E-mail: guoxing1958@sina.com

Dr Xiao-Dong Xie, Department of Operation Room, General Hospital of Shenyang Military Region, Shenyang 110001, P.R. China E-mail: than1984@aliyun.com

${ }^{*}$ Contributed equally

Key words: SHP2, laryngeal cancer, proto-oncogene, signal transduction interference of SHP2 expression can serve as a novel strategy for laryngeal cancer treatment.

\section{Introduction}

The overall incidence of laryngeal cancer has remarkably increased in recent decades and has become the second most common cancer of the respiratory system in China $(1,2)$. In the United States, over 13,000 new cases were detected and diagnosed in 2007 (3). The establishment of organ preservation approaches in recent years has improved the life quality of early cancer patients. However, the survival rate of patients with laryngeal cancer has decreased compared with the survival rate of patients with all other types of cancers as a whole (4). Therefore, the molecular pathogenesis of laryngeal cancer must be elucidated and novel strategies for treating laryngeal cancer must be developed.

The Src homologue phosphotyrosine phosphatase 2 (SHP2) is an intracellular tyrosine phosphatase with two tandem repeated Src homology 2 domains and has emerged as a major regulator of receptor tyrosine kinase, cytokine receptor, and hormone signaling $(5,6)$. Constitutively activating mutations in human gene PTPN11 encoding for SHP2 have been detected in almost $50 \%$ of Noonan syndrome patients who are at high risk for juvenile myelomonocytic leukemia. Somatic mutations resulting in constitutively active SHP2 have also been found in several types of leukemia (7). Notably, SHP2 and its binding scaffolding/adaptor protein Gab2 are overexpressed in a significant portion of breast cancer patients to promote mammary cell hyperproliferation (8). Furthermore, SHP2 is a major target of Helicobacter pylori CagA, which plays a key role in the pathogenesis of gastric carcinoma by activating SHP2 in a tyrosine phosphorylation-dependent manner $(9,10)$. Collectively, these findings indicate the involvement of SHP2 in the initiation and development of a variety of human malignancies (11). Therefore, PTPN11 is proposed to be the first proto-oncogene that encodes a tyrosine phosphatase (12).

In this study, we investigated the role of SHP2 in laryngeal cancer and its correlation with the prognosis of laryngeal cancer patients. Our findings provided a basis for 
the development of SHP2-targeted laryngeal cancer prevention and therapy.

\section{Materials and methods}

Ethics statement. All patients were from China and received treatment in the First Hospital of China Medical University. All clinical investigations were approved by the China Medical University Ethics Committee, and all patients involved in this study signed pertinent consent forms.

All animal studies were approved by the China Medical University Ethics Committee, and all animal experiments were conducted in the Experimental Animal Center of China Medical University according to International Guiding Principles for Biomedical Research Involving Animals. All animals were housed in virus-free facilities and maintained in a temperature and light (12/12-h light/dark cycle)-controlled environment. Mice were permitted ad libitum access to water and standard chow.

Animal experiments. For the growth assay, 18 eight-week-old male nude mice were divided into two groups and inoculated with Hep2 Control/siSHP2 cells $\left(2 \times 10^{6}\right)$. All mice were sacrificed by anesthesia overdose 8 weeks after inoculation. Tumors were measured by a Vernier caliper, weighed, and photographed. A portion of tumor tissues was collected, fixed in $10 \%$ formaldehyde, and embedded in paraffin for tumor pathological analysis.

Patients and microarray tissue samples. All human laryngeal tissues were obtained from surgical resection specimens of laryngeal cancer patients in China Medical University (Shenyang, China). For prognostic analysis, a tissue microarray was made from 112 laryngeal cancer samples with corresponding peritumor tissues collected from laryngeal cancer patients who underwent curative resection in China Medical University (Shenyang, China) from September 2005 to May 2012. For real-time PCR analysis, another 68 laryngeal cancer and corresponding peritumor frozen tissues were collected.

Immunohistochemistry and tissue microarray analysis. IHC analysis of tumor sections and tissue microarray were performed using the antibodies listed in Table I. The sections were incubated with primary antibodies at $4^{\circ} \mathrm{C}$ overnight, followed by horseradish peroxidase-conjugated secondary antibody at $37^{\circ} \mathrm{C}$ for $30 \mathrm{~min}$. The sections were then incubated with diaminobenzidine and counterstained with hematoxylin for detection. Assessment of tissue microarray staining was based on the percentage of positively stained cells, and staining intensity was measured by Image Scope (Aperio Technologies, Inc.) software. The expression levels of SHP2, p-Raf, p-MEK, and p-Erk in all 112 pairs of samples (cancer tissues and corresponding non-cancer adjacent tissues) were quantified (13). The ratio of SHP2, p-Raf, p-MEK, and p-Erk expression between each tumor and corresponding non-cancer surrounding tissue was calculated.

Cell lines and recombinant virus. Laryngeal cancer cell line Hep2 was purchased from the Cell Bank of Type Culture
Table I. Information for antibodies.

\begin{tabular}{lll}
\hline Name & Isotype & \multicolumn{1}{c}{ Company } \\
\hline p-MEK1/2 & Rabbit & Cell Signaling Technology \\
MEK1/2 & Rabbit & Cell Signaling Technology \\
GAPDH & Mouse & Santa Cruz Biotechnology, Inc. \\
p-Erk1/2 & Mouse & Cell Signaling Technology \\
Erk1/2 & Rabbit & Cell Signaling Technology \\
p-Raf & Rabbit & Santa Cruz Biotechnology, Inc. \\
Raf & Rabbit & Santa Cruz Biotechnology, Inc. \\
SHP2 & Rabbit & Cell Signaling Technology \\
\hline
\end{tabular}

Table II. Sequence of primers for real-time PCR.

\begin{tabular}{ll}
\hline Primer & \multicolumn{1}{c}{ Sequence $\left(5^{\prime} \rightarrow 3^{\prime}\right)$} \\
\hline Shp2 forward primer & CTGCCTCCACACCAGTGATA \\
Shp2 reverse primer & GGAGCCTGAGCAAGGAGC \\
18s forward primer & CGGCTACCACATCCAAGGAA \\
18s reverse primer & GCTGGAATTACCGCGGCT \\
c-Jun forward primer & ACCGACGAGCAGGAGGGCTT \\
c-Jun reverse primer & CAGCGCACCCGGGTTGAAGT \\
c-myc forward primer & GGCCGCTGCCAAACTGGTCT \\
c-myc reverse primer & TGGGCGAGCTGCTGTCGTTG
\end{tabular}

Collection of the Chinese Academy of Sciences. Lentivirus expressing siSHP2 or control was generated using Lenti-X Expression system (Clontech Laboratories, Inc.).

Cell proliferation assay. Hep2 siSHP2 and their control cells $\left(1 \times 10^{3}\right)$ were cultured in 96-well plates for various time periods. ATP activity was measured using a Cell Counting Kit-8 (Dojindo, Kumamoto, Japan) with a Synergy 2 microplate reader following the manufacturer's instructions. The results are presented as proliferation indices relative to control cells.

Real-time PCR. The original amount of specific transcripts was measured by real-time PCR using an ABI PRISM 7300 sequence detector (Applied Biosystems). The primer sequences are provided in Table II.

Western blot analysis. The Hep2 cell extract was separated on polyacrylamide-SDS gels, transferred, and probed with a specific primary antibody. The protein band, specifically bound to the primary antibody, was detected using an IRDye 800CW-conjugated secondary antibody and LI-COR imaging system (LI-COR Biosciences). The manufacturer information of the primary antibodies is provided in Table I.

Statistical analysis. Overall survival was defined as the interval between the dates of surgery and death. All statistical analyses were performed with SPSS version 18.0 software. The $\chi^{2}$ test or Fisher's exact test was used to compare qualitative variables, whereas continuous variables were compared 
A

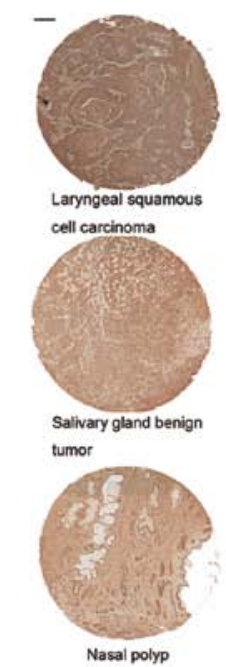

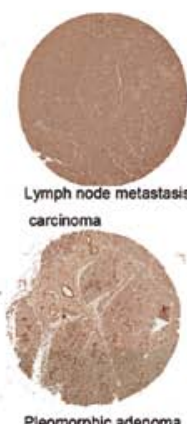

Pleomorphic adenoma

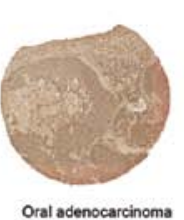

B
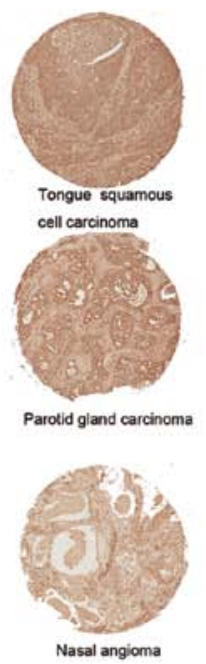

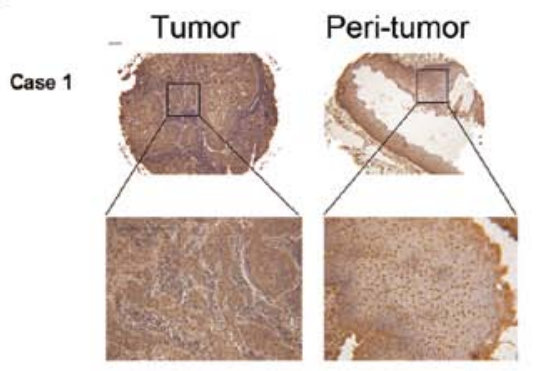

Case 2
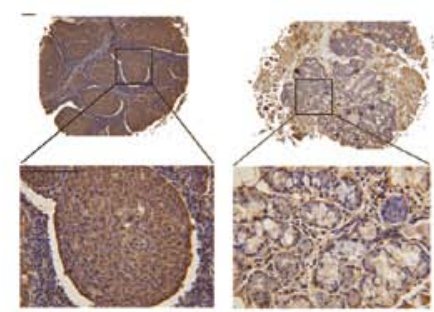

D
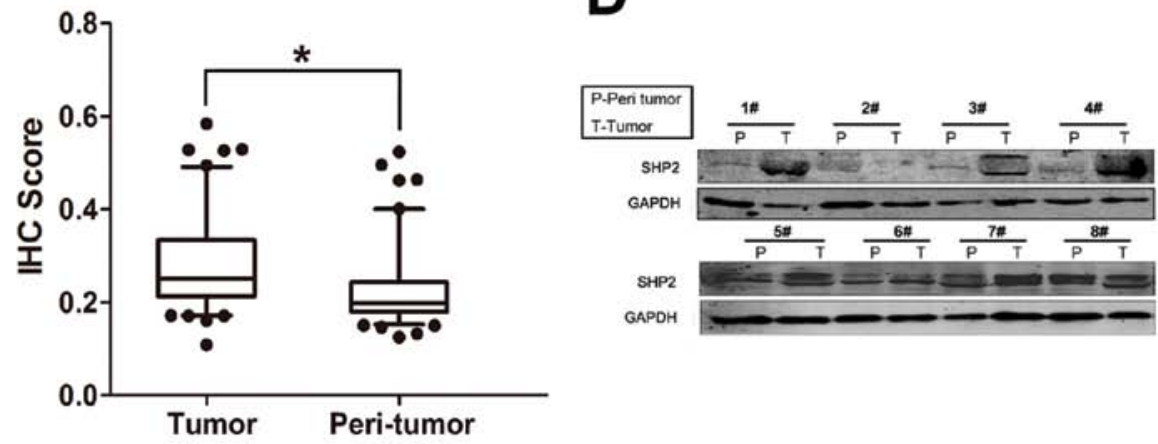

E

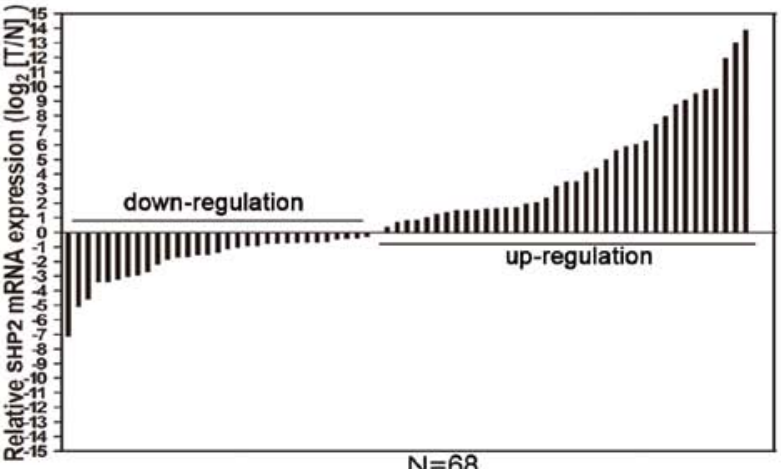

Figure 1. SHP2 expression is upregulated in laryngeal cancer compared with other head and neck tumors. (A) Representative view of immunohistochemical staining of SHP2 in a tissue array containing different head and neck tumor samples. Black scale bars, $100 \mathrm{~mm}$. (B and C) Immunohistochemical staining score of SHP2 expression from a tissue array contained 112 laryngeal cancer samples relative to correspoinding non-cancer tissues $\left(\mathrm{n}=112,{ }^{*} \mathrm{P}<0.05\right)$ and the representative view from tissue microarray. Black scale bars, $100 \mathrm{~mm}$. Micrograph indicates the amplified morphology of tumor tissue. (D) Representative western blot analysis results showing the expression of SHP2 protein in tumor tissue (T) and corresponding peritumor tissue (P) from 8 laryngeal cancer patients. (E) RT-PCR analysis of SHP2 mRNA expressions in laryngeal cancer patients $(n=68)$.

using Student's t-test or Mann-Whitney test for variables with abnormal distribution. Survival curves were calculated using the Kaplan-Meier method and compared with a log-rank test. The Cox proportional hazards model was used to determine the independent factors affecting survival based on variables selected by univariate analysis. $\mathrm{P}<0.05$ was considered statistically significant.

\section{Results}

SHP2 expression is upregulated in laryngeal cancer compared with other head and neck tumors. To explore the role of SHP2 in head and neck tumor, we first evaluated SHP2 expression in various head and neck tumors. Fig. 1A shows a tissue array containing different head and neck tumor samples 
Table III. Clinicopathological characteristics.

\begin{tabular}{|c|c|c|c|}
\hline \multirow[b]{2}{*}{ Variable } & \multicolumn{3}{|c|}{ Number and value (range) } \\
\hline & $\begin{array}{l}\text { SHP2-high } \\
\text { expression } \\
(\mathrm{n}=56)\end{array}$ & $\begin{array}{c}\text { SHP2-low } \\
\text { expression } \\
(\mathrm{n}=56)\end{array}$ & P-value \\
\hline $\begin{array}{l}\text { Median age } \\
\text { (range, years) }\end{array}$ & $63(40-85)$ & $64(58-79)$ & \\
\hline \multicolumn{4}{|l|}{ Gender } \\
\hline Male & $45(47.9 \%)$ & $49(52.1 \%)$ & 0.441 \\
\hline Female & $11(61.1 \%)$ & $7(38.9 \%)$ & \\
\hline \multicolumn{4}{|l|}{ Location $^{\mathrm{a}}$} \\
\hline Supraglottic & $35(60.3 \%)$ & $23(39.7 \%)$ & 0.027 \\
\hline Glottic & $17(35.4 \%)$ & $31(64,6 \%)$ & \\
\hline Subglottic & $4(66.7 \%)$ & $2(33.3 \%)$ & \\
\hline \multicolumn{4}{|l|}{ Tumor stage $^{a}$} \\
\hline $\mathrm{T} 1$ & $2(18.2 \%)$ & $9(81.8 \%)$ & 0.000 \\
\hline $\mathrm{T} 2$ & $8(81.0 \%)$ & $34(19.0 \%)$ & \\
\hline $\mathrm{T} 3$ & $33(78.6 \%)$ & $9(21.4 \%)$ & \\
\hline $\mathrm{T} 4$ & $13(23.5 \%)$ & $4(76.5 \%)$ & \\
\hline \multicolumn{4}{|l|}{ Nodal stage $^{a}$} \\
\hline No & $37(43.0 \%)$ & $49(57.0 \%)$ & 0.023 \\
\hline N1 & $5(83.3 \%)$ & $1(16.7 \%)$ & \\
\hline $\mathrm{N} 2 / \mathrm{N} 3$ & $14(70.0 \%)$ & $6(30.0 \%)$ & \\
\hline \multicolumn{4}{|l|}{ Tumor diameter $(\mathrm{cm})^{\mathrm{a}}$} \\
\hline$\leq 3$ & $29(38.7 \%)$ & $46(61.3 \%)$ & 0.001 \\
\hline$>3$ & $27(73.0 \%)$ & $10(27.0 \%)$ & \\
\hline \multicolumn{4}{|l|}{ Growth pattern ${ }^{\mathrm{a}}$} \\
\hline Exophytic & $28(39.4 \%)$ & $43(60.6 \%)$ & 0.001 \\
\hline Expansive & $7(46.7 \%)$ & $8(53.3 \%)$ & \\
\hline Ulcerative & $21(80.8 \%)$ & $5(19.2 \%)$ & \\
\hline \multicolumn{4}{|l|}{ Histological grade } \\
\hline Well & $37(52.9 \%)$ & $33(42.1 \%)$ & 0.271 \\
\hline Moderately & $11(37.9 \%)$ & $18(62.1 \%)$ & \\
\hline Poorly & $8(61.5 \%)$ & $5(38.5 \%)$ & \\
\hline \multicolumn{4}{|l|}{ Surgery ${ }^{\mathrm{a}}$} \\
\hline Partial laryngectomy & $17(34.7 \%)$ & $32(65.3 \%)$ & 0.007 \\
\hline Total laryngectomy & $39(61.9 \%)$ & $24(38.1 \%)$ & \\
\hline \multicolumn{4}{|l|}{ Smoking index } \\
\hline None & $6(66.7 \%)$ & $3(33.3 \%)$ & 0.453 \\
\hline $0-400$ & $12(42.9 \%)$ & $16(57.1 \%)$ & \\
\hline$>400$ & $38(50.7 \%)$ & $37(49.3 \%)$ & \\
\hline \multicolumn{4}{|l|}{ Alcohol abuse } \\
\hline None & $28(57.1 \%)$ & $21(42.9 \%)$ & \\
\hline Mild & $4(30.8 \%)$ & $9(69.2 \%)$ & 0.223 \\
\hline Heavy & $24 \quad(48 \%)$ & $26(52.0 \%)$ & \\
\hline
\end{tabular}

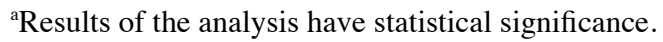

subjected to immunohistochemical staining. SHP2 expression was more elevated in laryngeal cancer and its neck lymph nodes than in other tumors. This finding prompted us to investigate whether SHP2 played an important role in laryngeal cancer tumorigenesis. A tissue array containing 112 laryngeal cancer samples relative to corresponding noncancer tissues in patients was used for immunohistochemical staining (Fig. 1B and C). The results were further confirmed by western blotting (Fig. 1D) and real-time PCR (Fig. 1E) analyses. We found that SHP2 expression was remarkably enhanced in laryngeal cancer.

SHP2 overexpression was associated with poor prognosis of laryngeal cancer. To investigate the clinical significance of SHP2 overexpression in laryngeal cancer, we further studied the role of SHP2 in laryngeal cancer at the molecular level. We found that SHP2 expression was correlated with the survival of laryngeal cancer patients. Higher SHP2 expression was associated with poorer prognosis of laryngeal cancer, whereas lower SHP2 expression corresponded to higher survival rates (Fig. 2A). We then examined the relationship between SHP2 expression in tumor tissues and the clinicopathological characteristics of the 112 patients (Table III). Correlation of regression analysis indicated that prognosis was correlated with several individual parameters, including SHP2 expression, tumor stage, nodal stage, tumor diameter, growth pattern, and surgery (Table IV). These individual parameters were further analyzed with a multivariate Cox proportional hazard model. Results indicated that tumor diameter, nodal stage, and SHP2 expression level were independent and significant factors that affected the survival of laryngeal cancer patients (Fig. 2C and Table IV). Among these factors, SHP2 expression level had a significant hazard ratio (HR) value for cumulative survival (HR, 2.837; 95\% confidence interval, 1.196-6.728; $\mathrm{P}=0.02$ ). We also analyzed the percentage of tumor diameters and nodal stage between low- and high-level SHP2 expression specimens. We found that patients with high-level SHP2 expression tended to have larger tumors and poorer nodal stages (Fig. 2D and E). These results suggested that SHP2 may function as a proto-oncogene.

Transfection of SHP2 into human laryngeal cancer cells promotes cell growth. To validate our findings that SHP2 acted as a proto-oncogene in laryngeal cancer, we used a human laryngeal cancer cell line to examine the effects of SHP2 expression on cell growth. The identification of Hep2 cell lines with over- or low-expressing SHP2, is shown in Fig. 3A. Using a cell-counting kit assay, we found that SHP2 influenced Hep2 (parental transfectant) cell growth (Fig. 3B). This result was further supported by the results of an anchorage-independent cell growth assay. Using a soft agar colony formation assay, we found that SHP2-overexpressing Hep2 (Hep2-SHP2) cells grew in the absence of anchorage, suggesting that increased SHP2 expression resulted in anchorage-independent cell growth (Fig. 3C). To determine the effects of elevated SHP2 expression on laryngeal cell growth in vivo, we established two stably transfected cell lines, namely, Hep2-siSHP2 and Hep2 control cells (Fig. 3A). Then, 2x106 Hep2-siSHP2 and Hep2 control cells were subcutaneously inoculated into nude mice for the xenograft assay. Fig. 3D shows that compared with 
Table IV. Univariate and multivariate analyses of the primary cohort.

\begin{tabular}{|c|c|c|c|c|}
\hline Variable & $\mathrm{n}$ & Time (months) $)^{\mathrm{b}}$ & P-value as univariate & $\mathrm{P}$-value as multivariate \\
\hline \multicolumn{5}{|l|}{ Shp2 expression ${ }^{\mathrm{a}}$} \\
\hline High & 56 & $43.857 \pm 21.855$ & 0.000 & 0.012 \\
\hline Low & 56 & $56.804 \pm 9.780$ & & \\
\hline \multicolumn{5}{|l|}{ Location } \\
\hline Supraglottic & 58 & $48.862 \pm 19.776$ & 0.167 & - \\
\hline Glottic & 48 & $53.958 \pm 13.043$ & & \\
\hline Subglottic & 6 & $35.500 \pm 27.783$ & & \\
\hline \multicolumn{5}{|l|}{ Tumor stage } \\
\hline $\mathrm{T} 1$ & 11 & $57.818 \pm 7.236$ & 0.000 & 0.357 \\
\hline $\mathrm{T} 2$ & 42 & $55.381 \pm 12.069$ & & \\
\hline $\mathrm{T} 3$ & 42 & $49.667 \pm 18.153$ & & \\
\hline $\mathrm{T} 4$ & 17 & $34.647 \pm 25.409$ & & \\
\hline \multicolumn{5}{|l|}{ Nodal stage $^{\mathrm{a}}$} \\
\hline N0 & 86 & $55.012 \pm 12.193$ & 0.000 & 0.020 \\
\hline $\mathrm{N} 1$ & 6 & $20.500 \pm 17.841$ & & \\
\hline $\mathrm{N} 2 / \mathrm{N} 3$ & 20 & $39.150 \pm 25.415$ & & \\
\hline \multicolumn{5}{|l|}{ Tumor diameter $(\mathrm{cm})^{\mathrm{a}}$} \\
\hline$\leq 3$ & 73 & $55.575 \pm 11.860$ & 0.000 & 0.042 \\
\hline$>3$ & 39 & $40.513 \pm 23.130$ & & \\
\hline \multicolumn{5}{|l|}{ Growth pattern } \\
\hline Exophytic & 71 & $52.225 \pm 16.272$ & 0.042 & 0.740 \\
\hline Expansive & 15 & $56.800 \pm 8.445$ & & \\
\hline Ulcerative & 26 & $41.423 \pm 23.449$ & & \\
\hline \multicolumn{5}{|l|}{ Histological grade } \\
\hline Well & 70 & $50.643 \pm 17.878$ & 0.477 & - \\
\hline Moderately & 29 & $52.724 \pm 15.736$ & & \\
\hline Poorly & 13 & $43.308 \pm 23.135$ & & \\
\hline \multicolumn{5}{|l|}{ Surgery } \\
\hline Partial laryngectomy & 49 & $55.102 \pm 12.754$ & 0.023 & 0.769 \\
\hline Total laryngectomy & 63 & $46.619 \pm 20.648$ & & \\
\hline \multicolumn{5}{|l|}{ Smoking index } \\
\hline None & 9 & $46.667 \pm 21.378$ & 0.458 & - \\
\hline $0-400$ & 28 & $48.893 \pm 20.977$ & & \\
\hline$>400$ & 75 & $51.307 \pm 16.619$ & & \\
\hline \multicolumn{5}{|l|}{ Alcohol abuse } \\
\hline None & 49 & $49.510 \pm 18.808$ & 0.545 & - \\
\hline Mild & 13 & $57.231 \pm 7.190$ & & \\
\hline Heavy & 50 & $49.340 \pm 19.125$ & & \\
\hline
\end{tabular}

${ }^{a}$ Results of the analysis were shown for the variables. ${ }^{b}$ Results were presented as mean \pm standard error.

the control cells, Hep2 cells with low-level SHP2 expression exhibited weaker tumorigenicity in nude mice both in terms of volume and weight (Fig. 3E and F). SHP2 expression in the tumors was identified by IHC staining. Representative images stained with hematoxylin and eosin are shown in Fig. 3G. Ki67 staining in tumors showed that SHP2 expression was lower in tissue sections from low SHP2 expression tumors than that in high SHP2 expression xenografts (Fig. 3G), suggesting 
A

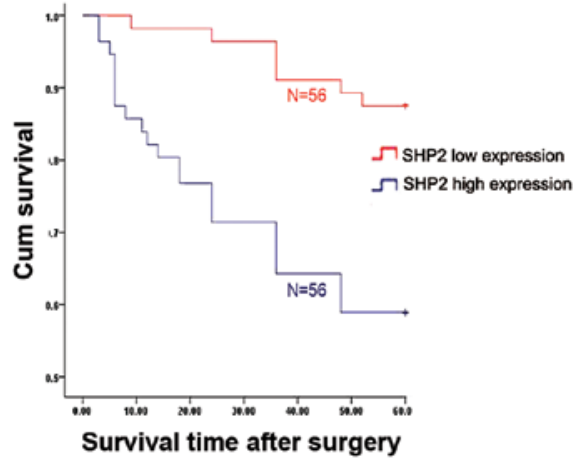

B

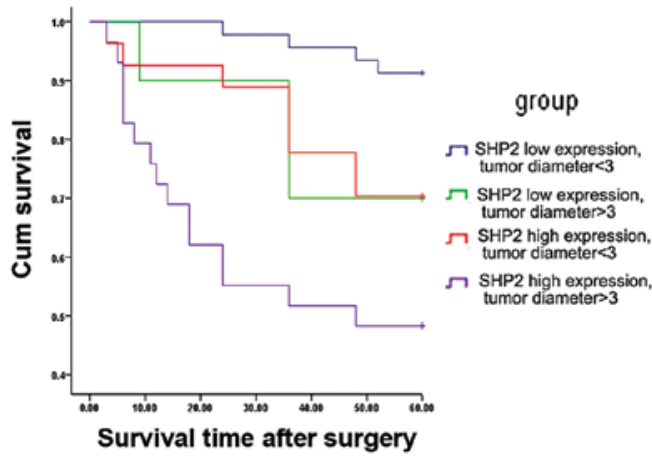

C

Survival

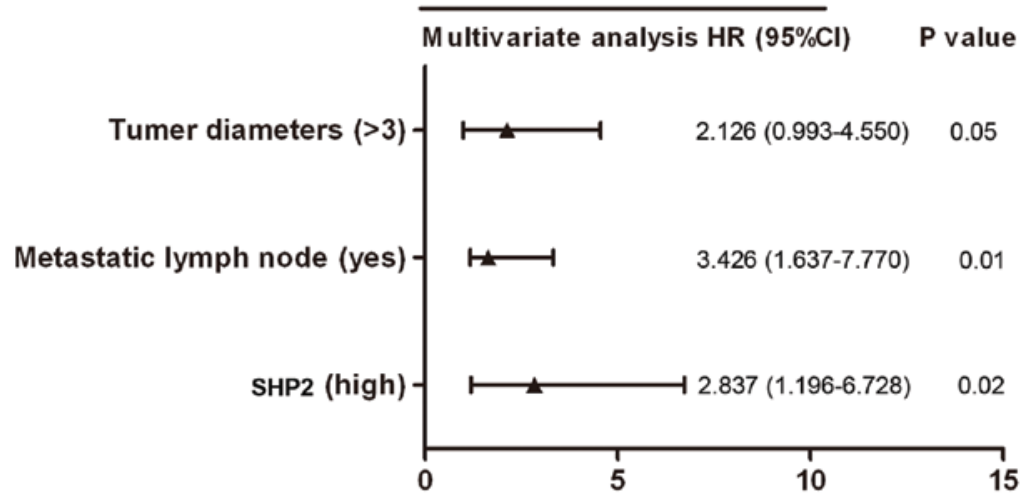

D

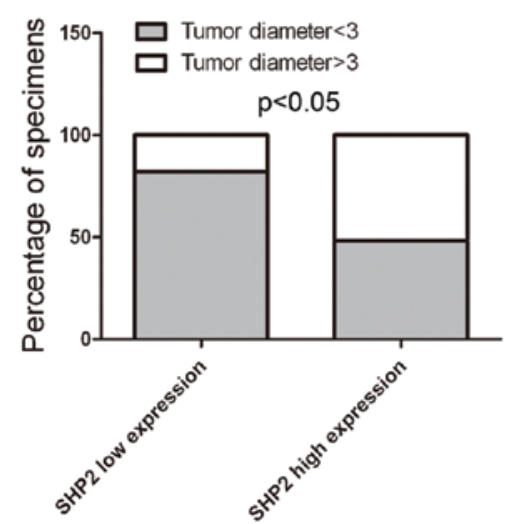

E

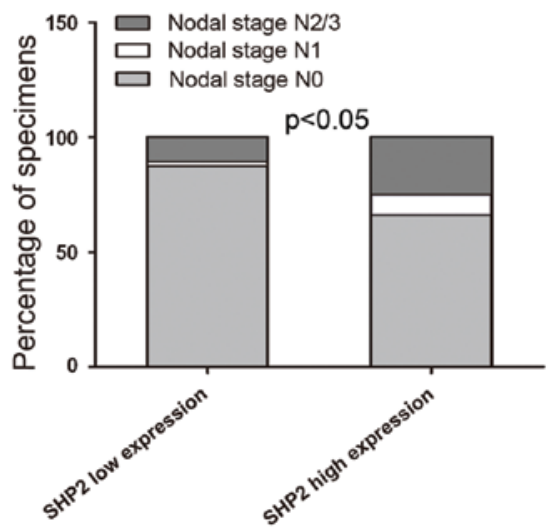

Figure 2. SHP2 overexpression is associated with poor prognosis of laryngeal cancer. (A) Laryngeal cancer patients were divided into an SHP2 'high' group (whose fold change of relative expression was higher than the median) and 'low' group (whose fold change of relative expression was lower than the median). The overall survival rates of the 112 laryngeal cancer patients were compared between the high and low SHP2 groups. (B) Overall survival between SHP2 expression level and different tumor diameter of laryngeal cancer patients. (C) Multivariate analysis of HRs for overall survival time. (D) The percentage of tumor diameters between SHP2 low expression and SHP2 high expression specimens ( $\mathrm{P}<0.05)$. (E) The percentage of nodal stage between SHP2 low expression and SHP2 high expression specimens $\left({ }^{*} \mathrm{P}<0.05\right)$.

an important role of SHP2 in Hep2 cell growth. Overall, the results in Fig. 3 suggested that SHP2 may be crucial to human laryngeal cancer growth.
SHP2 promotes laryngeal cancer growth by activating the Ras/Raf/MAPKs signaling pathway. To identify the molecular mechanism of laryngeal cancer growth promoted 
A
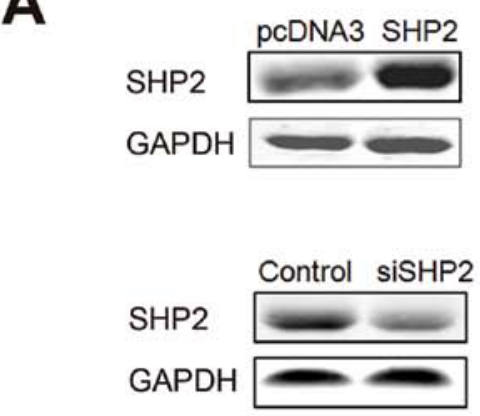

C

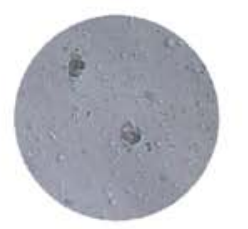

Hep2-pcDNA3

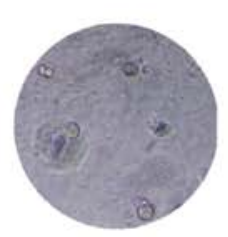

Hep2-SHP2
B
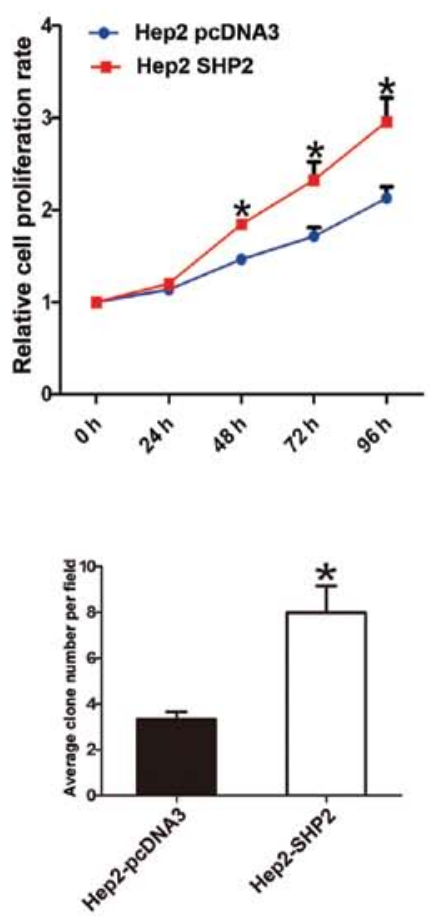

E

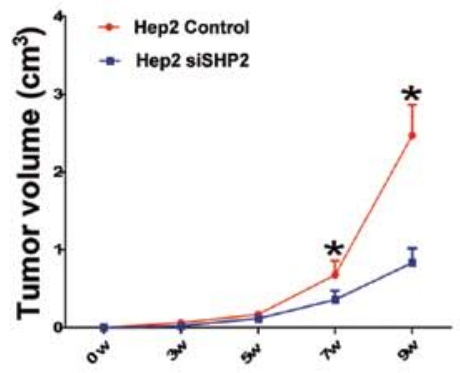

HE
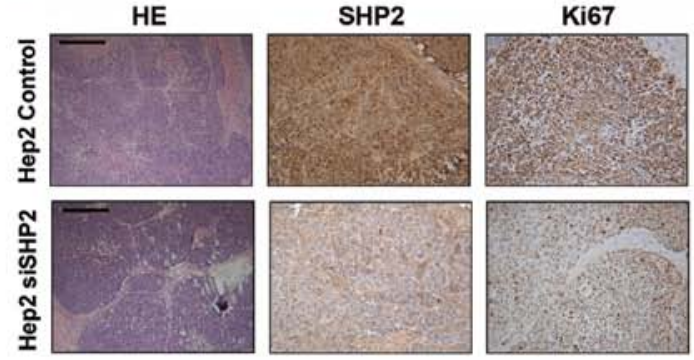

Figure 3. Transfection of SHP2 into human laryngeal cancer cells promotes cell growth. (A) The identification of Hep2 cell lines in parental transfected SHP2 overexpression plasmid and the pcDNA3 plasmid and identification of Hep2 stably transfected cell lines, Hep2-siSHP2 and Hep2 control cells. (B) Proliferation of Hep2-SHP2 and the control cells in different time-points. (C) For clone formation assay, Hep2-SHP2 or their control cells were seeded into a 96-well plate precoated with Matrigel and cultured for 7 days. Relative clones were calculated. Representative images were given bars representing SEM. ${ }^{*} \mathrm{P}<0.05$. (D) Hep2-siSHP2 and Hep2 control cells $\left(2 \times 10^{6}\right)$ were subcutaneously inoculated into nude mice for xenografts assay. Images for tumors of xenografts are shown. (E and F) Tumor volume and weight of xenografts were measured ( $\mathrm{P}<0.05)$. (G) Representative images of $\mathrm{H}$ and E-stained and immunohistochemical staining of SHP2 and Ki-67 in tissue sections from tumor xenografts. Black scale bars, $100 \mu \mathrm{m}$.

by SHP2, we assessed the expression profile of related genes in SHP2-expressing cells and analyzed the relevant regulatory signaling pathways. The Ras/Raf/MAPK signaling pathway is a predominant regulatory mechanism activated by SHP2 in leukemia (12). Therefore, the phosphorylation levels of Raf, MEK, and Erk were examined to determine whether 
A
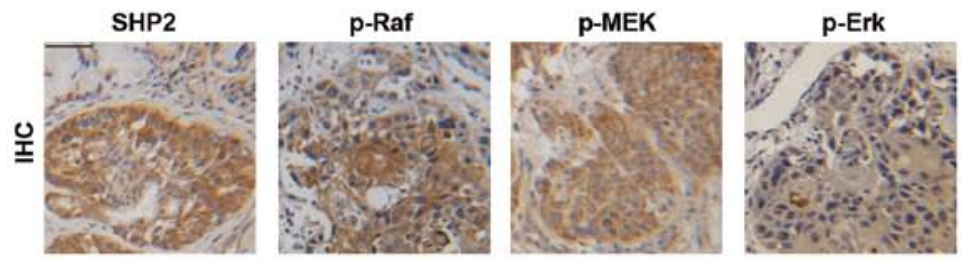

B

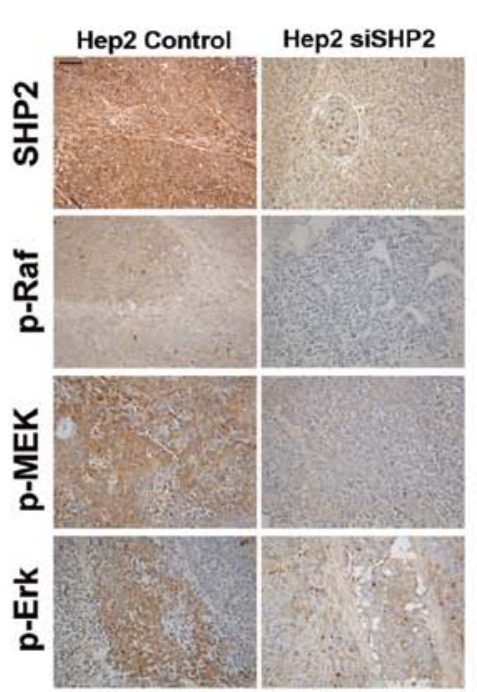

C

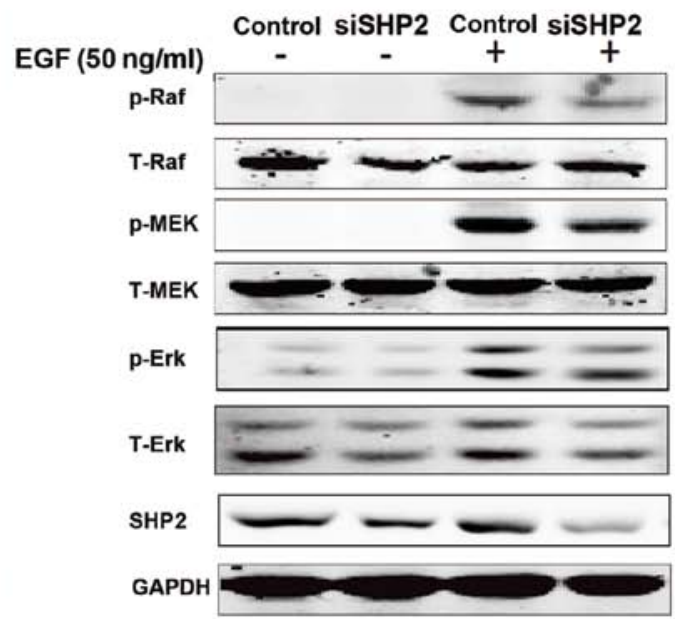

D

E
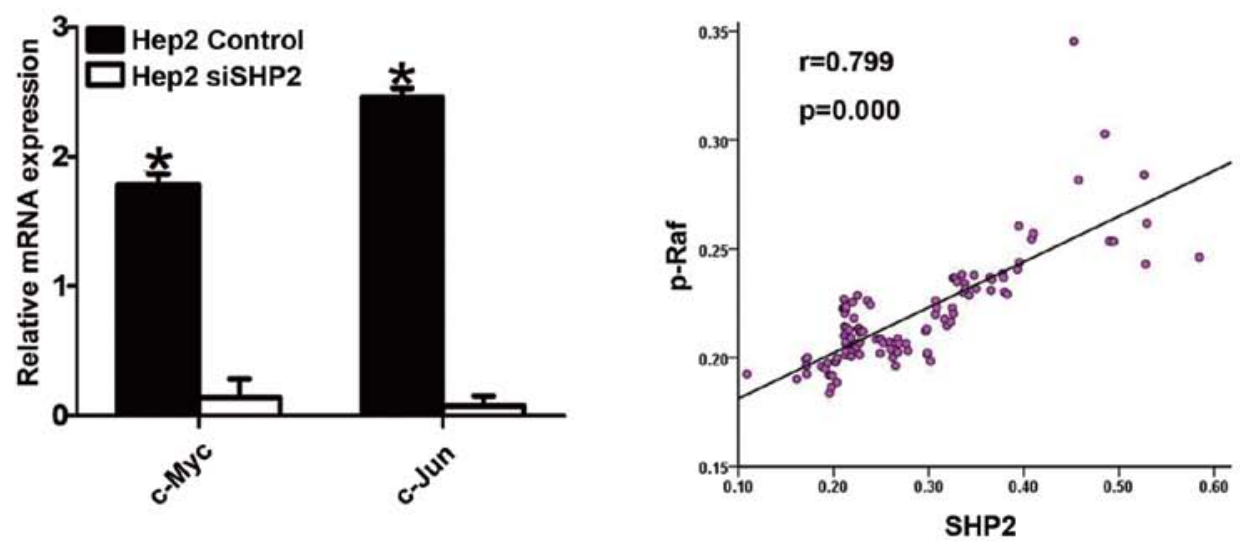

$\mathbf{F}$
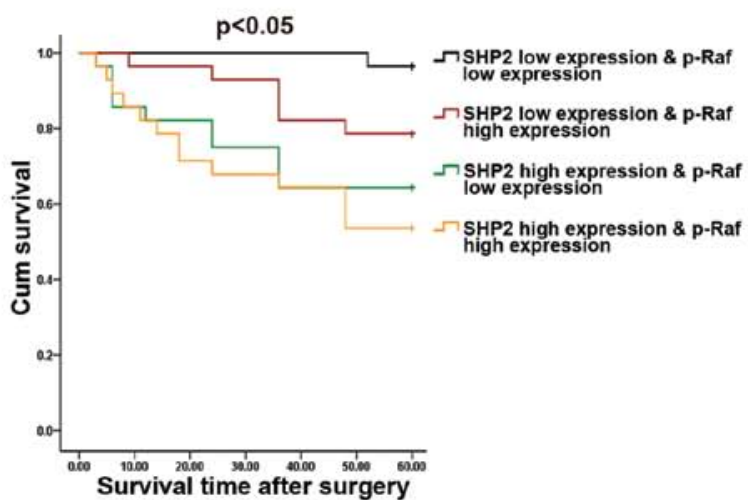

Figure 4. SHP2 promotes laryngeal cancer growth by activating the Ras/Raf/MAPKs signaling pathway. (A) Representative images of immunohistochemical staining of SHP2, p-Raf, p-MEK and p-Erk in tissue sections from laryngeal cancer patients. Black scale bars, $100 \mu \mathrm{m}$. (B) Representative images of immunohistochemical staining of SHP2, p-Raf, p-MEK and p-Erk in tissue sections from tumors of xenografts. Black scale bars, $100 \mu \mathrm{m}$. (C) Hep2-siSHP2 and Hep2 control stably transfected cells were subjected to western blot analysis after stimulated with EGF ( $50 \mathrm{ng} / \mathrm{ml})$. (D) Quantitative RT-PCR was performed to determine the mRNA levels of c-Jun and c-myc in Hep2-siSHP2 and Hep2 control cells as indicated. (E) The relationship (Pearson $\mathrm{r}=0.799$, P $<0.001)$ between SHP2 and p-Raf IHC score analyzed among the 112 patients by tissue microarray. (F) Laryngeal cancer patients were divided into 4 groups, SHP2 high expression and p-Raf high expression group, SHP2 high expression and p-Raf low expression group, SHP2 low expression and p-Raf high expression group, SHP2 low expression and p-Raf low expression group, the overall survival rates of the 112 laryngeal cancer patients were compared among different groups. 
phosphorylation was required in SHP2-mediated laryngeal cancer growth. Fig. 4A shows that the phosphorylation levels of Raf, MEK and Erk were significantly upregulated in tissues of laryngeal cancer patients. In the subcutaneous xenografts, we confirmed this result in Hep2-siSHP2 cells (Fig. 4B). Furthermore, the phosphorylation levels of Raf, MEK and Erk were significantly downregulated after stimulation with EGF $(50 \mathrm{ng} / \mathrm{ml})$ in low SHP2-expressing Hep2 cells compared with the levels in the control cells (Fig. 4C). Downregulation of the downstream transcription factors of this pathway such as c-Jun and c-Myc was also observed in low SHP2-expressing cells (Fig. 4D). SHP2 was closely correlated with p-Raf in the 112 laryngeal cancer tissues samples $(r=0.799, p<0.001)$ (Fig. 4E). In addtion, the combination analysis of SHP2 expression and p-Raf expression could assess the overall survival rates of 112 laryngeal cancer patients more accurate (Fig. 4F).

\section{Discussion}

Laryngeal carcinoma accounts for $90 \%$ of carcinomas found in the head and neck region. This cancer is often fatal despite current treatment protocols involving surgery, radiation and chemotherapy $(14,15)$. Even with new treatment protocols, no significant improvement in 5-year overall survival rates has been observed (15). Novel therapeutic strategies specifically targeting growth pathways utilized by tumor cells have shown great potential in treating this disease.

SHP2 encoded by the PTPN11 gene is a protein tyrosine phosphatase (PTP) containing two tandem repeated Src homology 2 domains in its $\mathrm{N}$-terminal region (15). In contrast to many other protein phosphatases, SHP2 promotes rather than inhibits cellular processes such as cell proliferation and motility (16). Germline gain-of-function mutations in PTPN11 have been reported in $50 \%$ of patients with Noonan syndrome, a developmental disorder characterized by facial abnormalities, short stature, congenital heart defects and increased risk of hematological malignancies, notably juvenile myelomonocytic leukemia (17). Known as the first bona fide PTP oncogenic regulator (12), SHP2 overexpression has been detected in numerous tumors, such as several types of leukemia and breast cancer $(18,19)$. SHP2 overexpression also triggers the activation of several cytoplasmic kinase cascade pathways, such as the Ras-Raf-Erk (20), JAK/STAT (21) and PI3K/AKT pathways (22). Moreover, studies on mouse genetics, gene silencing, and sequencing reveal a broad role of SHP2 in cell fate and tumor development $(18,19,23,24)$.

In the present study, we showed that SHP2 expression increased at both mRNA and protein levels in laryngeal cancer, and this elevated expression was associated with malignant clinicopathological characteristics. The correlation between SHP2 expression and surgical outcomes was further investigated in a retrospective study of 112 laryngeal cancer patients. We found that tumor survival rates substantially differed between patients with high- and low-level expressions of SHP2 in tumor tissues. Multivariate analysis revealed that SHP2 expression was an independent and significant risk factor affecting survival rate after curative resection, with the greatest HR value for survival. More importantly, SHP2 overexpression showed enhanced accuracy in predicting surgical outcome in patients with laryngeal cancer at early stages. To gain insight into the mechanism regulating laryngeal cancer tumorigenesis, we established a stable cell line expressing lowlevel SHP2 by recombinant lentivirus. We further found that SHP2 could promote laryngeal cancer cell growth and tumor formation, and under the stimulation of EGF (50 ng/ml), SHP2 could activate the Ras/Raf/Erk pathway which play an important role in tumor growth $(25,26)$. Moreover, SHP2 expression is closely correlated with p-Raf expression in laryngeal cancer, and the combination analysis of SHP2 expression and p-Raf expression would help to assess the prognosis of laryngeal cancer patients. These data strongly suggested that SHP2 had clinical significance by targeting the SHP2-activated signaling pathway in the individualized therapy of laryngeal cancer patients. The data also provided new insight into the prognosis of laryngeal tumorigenesis and the individualized therapy of patients. Therefore, we believe that SHP2 functioned as a proto-oncogene, and its elevated expression activated the Ras/ Raf/Erk pathway and contributed to complicated malignant progression.

In conclusion, methods traditionally used to determine the clinical management of laryngeal cancer, one of the most common malignancies in the head and neck, are now considered inadequate for current clinical protocols (27). Although attempts have been made to improve the prediction of patient outcome using new markers such as p53, p21, and EGFR (28-30), results are not satisfactory. However, SHP2 as a novel regulator of laryngeal cancer may have an important role in laryngeal cancer tumorigenesis through the Ras/Raf/MEK/Erk signaling pathway and may serve as a valuable prognostic biomarker and potential therapeutic target. Thus, our study revealed a novel function of SHP2 and the molecular mechanism by which SHP2 promoted laryngeal cancer tumorigenesis.

\section{Acknowledgements}

The authors would like to thank Ying Li for her technical assistance and sincerely thank G.S. Feng and H.Y. Wang for their selfless guidance. This study was supported by grants from National Natural Science Foundation of China, 81000931; Medical Peak Construction Special Fund of Liaoning Province, 4010218; Ministry of Science and Technology key program, 2012ZX10002-009.

\section{References}

1. Li XY, Guo X, Feng S, et al: Relationship between a family history of malignancy and the incidence of laryngeal carcinoma in the Liaoning province of China. Clin Otolaryngol 34: 127-131, 2009.

2. Jemal A, Siegel R, Ward E, Murray T, Xu J and Thun MJ: Cancer statistics, 2007. CA Cancer J Clin 57: 43-66, 2007.

3. Loyo M and Pai SI: The molecular genetics of laryngeal cancer. Otolaryngol Clin North Am 41: v657-v672, 2008.

4. Hoffman HT, Porter K, Karnell LH, et al: Laryngeal cancer in the United States: changes in demographics, patterns of care, and survival. Laryngoscope 116: 1-13, 2006.

5. Neel BG, Gu H and Pao L: The 'Shp'ing news: SH2 domaincontaining tyrosine phosphatases in cell signaling. Trends Biochem Sci 28: 284-293, 2003.

6. Lai LA, Zhao C, Zhang EE and Feng GS: The Shp-2 tyrosine phosphatase. In: Protein Phosphatases. Arino J and Alexander D (eds). Springer-Verlag, Berlin, Heidelberg, pp275-299, 2004.

7. Tartaglia M and Gelb BD: Germ-line and somatic PTPN11 mutations in human disease. Eur J Med Genet 48: 81-96, 2005.

8. Bentires-Alj M, Gil SG, Chan R, et al: A role for the scaffolding adapter GAB2 in breast cancer. Nat Med 12: 114-121, 2006. 
9. Higuchi M, Tsutsumi R, Higashi $\mathrm{H}$ and Hatakeyama $\mathrm{M}$ Conditional gene silencing utilizing the lac repressor reveals a role of SHP-2 in cagA-positive Helicobacter pylori pathogenicity. Cancer Sci 95: 442-447, 2004.

10. Higashi H, Tsutsumi R, Muto S, et al: SHP-2 tyrosine phosphatase as an intracellular target of Helicobacter pylori CagA protein. Science 295: 683-686, 2002.

11. Takahashi A, Tsutsumi R, Kikuchi I, et al: SHP2 tyrosine phosphatase converts parafibromin/Cdc73 from a tumor suppressor to an oncogenic driver. Mol Cell 43: 45-56, 2011.

12. Chan RJ and Feng GS: PTPN11 is the first identified proto-oncogene that encodes a tyrosine phosphatase. Blood 109: 862-867, 2007.

13. Wen W, Han T, Chen C, et al: Cyclin G1 expands liver tumorinitiating cells by Sox 2 induction via Akt/mTOR signaling. Mol Cancer Ther 12: 1796-1804, 2013.

14. Broich G, Lavezzi AM, Biondo B and Pignataro LD: PCNA - a cell proliferation marker in vocal cord cancer. Part II: recurrence in malignant laryngeal lesions. In Vivo 10: 175-178, 1996.

15. Dobrossy L: Epidemiology of head and neck cancer: magnitude of the problem. Cancer Metastasis Rev 24: 9-17, 2005

16. Manes S, Mira E, Gomez-Mouton C, Zhao ZJ, Lacalle RA and Martinez AC: Concerted activity of tyrosine phosphatase SHP-2 and focal adhesion kinase in regulation of cell motility. Mol Cel Biol 19: 3125-3135, 1999.

17. Tartaglia M, Mehler EL, Goldberg R, et al: Mutations in PTPN11, encoding the protein tyrosine phosphatase SHP-2, cause Noonan syndrome. Nat Genet 29: 465-468, 2001.

18. Aceto N, Sausgruber N, Brinkhaus H, et al: Tyrosine phosphatase SHP2 promotes breast cancer progression and maintains tumorinitiating cells via activation of key transcription factors and a positive feedback signaling loop. Nat Med 18: 529-537, 2012.

19. Grossmann KS, Rosario M, Birchmeier C and Birchmeier W: The tyrosine phosphatase Shp2 in development and cancer. Adv Cancer Res 106: 53-89, 2010.

20. Cunnick JM, Meng S, Ren Y, et al: Regulation of the mitogenactivated protein kinase signaling pathway by SHP2. J Biol Chem 277: 9498-9504, 2002.
21. Dittrich A, Quaiser T, Khouri C, Gortz D, Monnigmann M and Schaper F: Model-driven experimental analysis of the function of SHP-2 in IL-6-induced Jak/STAT signaling. Mol Biosyst 8: 2119-2134, 2012

22. Tseng PC, Huang WC, Chen CL, et al: Regulation of SHP2 by PTEN/AKT/GSK-3beta signaling facilitates IFN-gamma resistance in hyperproliferating gastric cancer. Immunobiology 217 926-934, 2012.

23. Chan G, Kalaitzidis D and Neel BG: The tyrosine phosphatase Shp2 (PTPN11) in cancer. Cancer Metastasis Rev 27: 179-192, 2008.

24. Feng GS: Shp2-mediated molecular signaling in control of embryonic stem cell self-renewal and differentiation. Cell Res 17: 37-41, 2007.

25. Wu R, Duan L, Ye L, et al: S100A9 promotes the proliferation and invasion of HepG2 hepatocellular carcinoma cells via the activation of the MAPK signaling pathway. Int J Oncol 42: 1001-1010, 2013

26. Chung EJ, Urick ME, Kurshan $\mathrm{N}$, et al: MEK1/2 inhibition enhances the radiosensitivity of cancer cells by downregulating survival and growth signals mediated by EGFR ligands. Int J Oncol 42: 2028-2036, 2013.

27. Pradier R, Gonzalez A, Matos E, et al: Prognostic factors in laryngeal carcinoma. Experience in 296 male patients. Cancer 71: 2472-2476, 1993

28. Jin YT, Kayser S, Kemp BL, et al: The prognostic significance of the biomarkers $\mathrm{p} 21^{\mathrm{WAF} 1 / \mathrm{CIP1}}$, p53, and bcl-2 in laryngeal squamous cell carcinoma. Cancer 82: 2159-2165, 1998.

29. Tandon S, Tudur-Smith C, Riley RD, Boyd MT and Jones TM A systematic review of $\mathrm{p} 53$ as a prognostic factor of survival in squamous cell carcinoma of the four main anatomical subsites of the head and neck. Cancer Epidemiol Biomarkers Prev 19: 574-587, 2010.

30. Fujii S, Uryu H, Akashi K, et al: Clinical significance of KRAS gene mutation and epidermal growth factor receptor expression in Japanese patients with squamous cell carcinoma of the larynx, oropharynx and hypopharynx. Int J Clin Oncol 118: 454-463, 2013. 